Article

\title{
Vulnerability Analysis of Urban Rail Transit Networks: A Case Study of Shanghai, China
}

\section{Daniel (Jian) Sun ${ }^{1,2}$, Yuhan Zhao ${ }^{2,3, *}$ and Qing-Chang Lu ${ }^{1}$}

1 State Key Laboratory of Ocean Engineering, School of Naval Architecture, Ocean \& Civil Engineering, Shanghai Jiao Tong University, Shanghai 200240, China; E-Mails: danielsun@sjtu.edu.cn (D.J.S.); qclu@sjtu.edu.cn (Q.-C.L.)

2 Transportation Research Center, School of Naval Architecture, Ocean and Civil Engineering, Shanghai Jiao Tong University, Shanghai 200240, China

3 Department of Management, Technology and Economics, ETH Zurich, CH-8093 Zurich, Switzerland

* Author to whom correspondence should be addressed; E-Mail: yuhzhao@ethz.ch; Tel.: +86-21-3420-6674; Fax: +86-21-3420-6197.

Academic Editor: Marc A. Rosen

Received: 29 March 2015 / Accepted: 30 April 2015 / Published: 28 May 2015

\begin{abstract}
Rail transit is developing rapidly in major cities of China and has become a key component of urban transport. Nevertheless, the security and reliability in operation are significant issues that cannot be neglected. In this paper, the network and station vulnerabilities of the urban rail transit system were analyzed based on complex network and graph theories. A vulnerability evaluation model was proposed by accounting metro interchange and passenger flow and further validated by a case study of Shanghai Metro with full-scale network and real-world traffic data. It is identified that the urban rail transit network is rather robust to random attacks, but is vulnerable to the largest degree node-based attacks and the highest betweenness node-based attacks. Metro stations with a large node degree are more important in maintaining the network size, while stations with a high node betweenness are critical to network efficiency and origin-destination (OD) connectivity. The most crucial stations in maintaining network serviceability do not necessarily have the highest passenger throughput or the largest structural connectivity. A comprehensive evaluation model as proposed is therefore essential to assess station vulnerability, so that attention can be placed on appropriate nodes within the metro system. The findings of this research are of both theoretical and practical significance for urban rail transit network design and performance evaluation.
\end{abstract}


Keywords: vulnerability analysis; urban rail transit networks; complex network theory; metro interchange; passenger flow

\section{Introduction}

In recent years, rapid urbanization and motorization in the major cities of China have resulted in a huge population explosion and private vehicle ownership, which consequently bring serious problems to traditional road transport, such as urban congestions and pollution, etc. Compared with road transport, urban rail transit, such as metro, light rail and regional railways, provide a variety of economic, social and environmental benefits [1]. Nevertheless, their security and stability in operation have become significant issues that cannot be neglected. In particular, network failures, outbursts of passenger flows, natural disasters and terrorist activities may cause the breakdown of stations or lines, affecting the overall efficiency of the rail networks [2]. In Shanghai, for example, on 27 October 2007, an electricity power failure led to the breakdown of Metro Line 1, Line 2 and Line 4 for more than one hour; on 22 December 2009, a power blackout of Metro Line 1 directly resulted in a two-train crash; on 27 September 2011, a rear-end accident on Metro Line 10 occurred between Yuyuan Garden and Laoximen stations. Moreover, on 14 September 2013, water intrusion caused signal failures for Metro Line 2 and Line 6, leading to a large number of passengers stranded at the stations for more than four hours. Compared with road network accidents, rail accidents not only cause traffic delays of the direct line(s), but also have a wider impact on passengers in other stations along the line(s) or even potential passengers, which generally generate a larger social impact.

With a history of more than 150 years, urban rail transit has attracted comparable more attention in the prevention of network failures and system disruptions [3-7]. In the recent ten years, graph and complex network theories have been introduced for analyzing the reliability and safety of urban rail transit networks. However, studies on this specific approach remain relatively limited. Del Río et al. [8] analyzed the resilience capabilities of underground systems and calculated the amount of backup capacity required to recover from system failures. Derrible and Kennedy [9] introduced robustness indicators corresponding to the characteristics of transit systems by investigating 33 metro systems throughout the world. Recommendations for improving the robustness of differently-sized metro networks were provided accordingly. De-Los-Santos et al. [10] proposed passenger robustness measures for rail transit networks under with and without bridging interruptions and verified the measures on the Madrid commuter system. Cadarso et al. [11] studied the disruption management problem of rapid transit rail networks and designed a two-step approach that combined an integrated optimization for the timetable and rolling stock by considering the passenger behavior.

It is worth mentioning that although the concepts of reliability, resilience and robustness are closely related to the general subject of vulnerability [12], these terms are different in research scopes. Transport vulnerability is largely a fundamental feature of the urban system and concerns the consequences and probabilities of system failures [13]. Therefore, the above-mentioned literature tried to provide various tools in addressing vulnerability-related problems, but did not look into the issue of vulnerability directly. 
Currently, vulnerability analysis of urban rail transit networks mainly refers to the research achievements in road network systems [13-16]. Gao and Shi [17] proposed an evaluation model of metro system invulnerability based on network topology and calculated the evaluation indices with matrix logic. Wang [18] constructed the topological model of the Beijing transit network and simulated the network efficiency under various attacks. Zhang et al. [19] measured the topological characteristics and functional properties of the Shanghai metro system. Nevertheless, these studies simplified the urban rail transit networks with graph theory and, therefore, lacked consideration of the significant properties of rail transit systems, such as the ability to transfer, etc.

Other approaches to vulnerability analysis were employed. Quan et al. [20] established an index system to assess the vulnerability of rainstorm water-logging in Shanghai Metro. Han et al. [21] analyzed urban mass transit accidents from three aspects, including interference, exposure and vulnerability. They regarded vulnerability as inherent defects of the system and established a theoretical safety insurance mechanism. Yuan et al. [22] studied the statistical data of metro network accidents and proposed the concepts of physical, structural and social vulnerabilities of metro system. These studies, unfortunately, lacked systematical analyses of urban rail transit networks, which may hinder accurate the definition, design and performance evaluation of the system, while these are particularly useful for the public transportation planners and practitioners.

To provide theoretical support to the planning and operation of urban rail transit networks, this study conducts a comprehensive analysis of the vulnerability of urban rail transit networks. The paper is organized as follows. In Section 2, urban rail transit networks were modeled with the proposed topological parameters, so that a systematical vulnerability evaluation model can be built up. In Section 3, vulnerability analyses of Shanghai Metro were carried out, with a case study based on the proposed evaluation model. Finally, the paper closes with conclusions and possible research directions in Section 4.

\section{Model Descriptions}

\subsection{Construction of Urban Rail Transit Networks}

A complex network model generally defines elements of the system as vertices, whose edges represent the interactions between vertices [23]. According to Angeloudis and Fisk's study on the 20 largest subways throughout the world [24], urban rail transit networks can be depicted as complex networks, which possess the characteristics of high connectivity, but low maximum vertex degree and have typical features of both small-world and scale-free categories.

Stations and metro lines are the basic components of the urban rail transit system. Based on complex network theory, stations can be virtualized into nodes of complex networks, while metro lines can be virtualized into edges to connect the nodes. As urban metros generally have two-way traffic, the rail transit network is viewed as an undirected graph $G=\langle V, E\rangle$, in which $V=\left\{v_{i} \mid i=1,2,3 \ldots N\right\}$ is the set of network nodes and $E=\left\{e_{i j} \mid v_{i}, v_{j} \in V\right\}$ is the set of network edges. $A=\left[a_{i j}\right]_{N \times N}$ is the network adjacency matrix, where $a_{i j}$ is defined as:

$$
a_{i j}=\left\{\begin{array}{l}
1,\left(v_{i}, v_{j}\right) \in E \\
0,\left(v_{i}, v_{j}\right) \notin E
\end{array}\right.
$$


In urban rail transit networks, degree $\left(D_{i}\right)$ is defined as the number of edges connecting with node $v_{i}$, and betweenness $\left(B_{i}\right)$ is defined as the number of shortest paths between any two nodes in the network passing node $v_{i}$. The two topological parameters depict the connectivity and the pressure of a given network, respectively.

Meanwhile, many urban rail transit lines do not allow passengers to transfer on the same platform. Therefore, transfer duration, including the walking time from one metro line to another and the waiting time for the next train, should be incorporated. In general, passengers psychologically prefer to choose routes with fewer interchanges, which largely affects their route preference [25] and has further impacts on the shortest path and network efficiency. To this end, transfer duration is quantified by assigning impedance to the interchange stations. A simplified example is presented in Figure 1a. Two lines, A-C-E and B-C-D, intersect each other, with $\mathrm{C}$ as the interchange station. As shown in Figure 1b, in the modified model, node $C$ is divided into two virtual nodes $\left(C_{1}\right.$ and $\left.C_{2}\right)$, and the two lines are separated, with only $\mathrm{C}_{1}-\mathrm{C}_{2}$ connected. The shortest path for origin-destination (OD) pair A-D changes into A-C $\mathrm{C}_{1}-\mathrm{C}_{2}-\mathrm{D}$, while the shortest path for OD pair A-E remains unchanged. Each interchange brings the corresponding walking and waiting time to the trip, and the transfer time is therefore quantified as the path length.

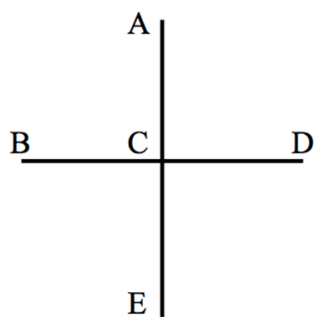

(a)

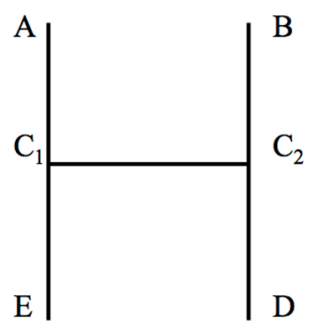

(b)

Figure 1. Method to quantify metro interchange: (a) original model; (b) modified model.

Then, the quantify algorithm is explained as follows:

(1) Each interchange station (with degree as $D_{i}$ ) is divided into $D_{i} / 2$ virtual nodes. These virtual nodes, connecting with each other, belong to separate metro lines, and the related network adjacency matrix can then be obtained.

(2) Input: the network adjacency matrix, including node names (virtual nodes that belong to one interchange station have the same node name $v_{i}$ ) and other parameters.

(3) Calculate the shortest paths based on the expanding network adjacency matrix with the Floyd algorithm [26] and obtain the corresponding matrix.

(4) For all paths, whose origins or destinations have the same node name $v_{i}$ (the same interchange station), find the shortest path, and delete the others. In Figure 1b, for example, OD pair A-C has two paths, $\mathrm{A}-\mathrm{C}_{1}$ and $\mathrm{A}-\mathrm{C}_{2}$, with lengths of one and two, respectively, and consequently, the longer path $A-C_{2}$ was deleted. Similarly, the paths $E_{-} C_{2}, B-C_{1}, D-C_{1}, C_{1}-D, C_{1}-B, C_{2}-E$ and $C_{2}-A$ were also removed.

(5) Each interchange station is divided into several virtual nodes to consider the interchange effort. Paths between these nodes need to be deleted, as they do not exist in reality. For example, $\mathrm{C}_{1}-\mathrm{C}_{2}$ and $\mathrm{C}_{2}-\mathrm{C}_{1}$ in Figure $1 \mathrm{~b}$ are removed as $\mathrm{C}_{1}$ and $\mathrm{C}_{2}$ denote the same interchange station.

(6) Output: the shortest path $\left(l_{\mathrm{ij}}\right)$ between any two stations $v_{i}$ and $v_{j}$ in the network. 


\subsection{Vulnerability Evaluation Model}

Vulnerability in traffic engineering is generally defined as "a susceptibility to incidents that may result in considerable reductions in network serviceability" [12], involving two components: (1) the probability that an event would happen and cause negative impacts; and (2) the negative consequences once the event has taken place. In this paper, urban rail transit networks were investigated from two aspects: network vulnerability and station vulnerability. Network vulnerability analysis consists of topological structure and functional ability and is carried out with attacking experiments. Node-based malicious attacks, generally considered as the most significant issue in network malfunctions, were studied and grouped into three types, namely the largest degree node-based attacks, the highest betweenness node-based attacks and random attacks.

\subsubsection{Topological Vulnerability}

Topological vulnerability is defined as network susceptibility to incidents, which may result in the reduction of structure connectivity. To this end, topological efficiency $(E)$, calculated as the mean of the reciprocal of each shortest path within the network, is used to evaluate network topological vulnerability as follows:

$$
E=\frac{1}{N(N-1)} \sum_{v_{i}, v_{j} \in V} \frac{1}{l_{i j}}
$$

where $N$ is the number of nodes in the network and $l_{\text {ij }}$ is the shortest path between stations $v_{i}$ and $v_{j}$. The index depicts the overall connectivity of the network, and a higher value of $E$ indicates that the network is more efficient in OD transferring.

\subsubsection{Functional Vulnerability}

Functional vulnerability is defined as network susceptibility to incidents that may result in a reduction in transport ability. Each node in the network is supposed to possess an initial functional ability of one. If a node is removed from the network (attacked or isolated), the functional ability reduces to zero. Based on this definition, network size was defined and used to evaluate network functional vulnerability. When a network is attacked, the dysfunctional nodes have to be removed from the network, which may induce some isolated nodes within the network. Consequently, network size is therefore defined as the total number of nodes remaining connected within the network.

To better analyze network transport ability, the concept of OD is introduced. When node $v_{i}$ is attacked, network efficiency generally decreases due to the increased system travel time; worst of all, some OD pairs would become unconnected, which indicates a dramatic reduction in transport ability. The connected OD ratio is therefore defined as the percentage of OD pairs remaining connected in the network, revealing the system robustness in maintaining network functional properties.

Previous literature $[19,27]$ used the largest connected cluster (LCC) to evaluate the functional vulnerability of urban rail transit networks, which was defined as the size of the largest sub-network after being attacked. However, this parameter is not suitable to be applied to urban rail transit networks, 
as trains may still be in operation on each sub-network, even if the network is divided into multiple isolated sub-networks.

\subsubsection{Station Vulnerability}

Based on these concepts, station vulnerability is further defined to evaluate the vulnerability of each station. When a station $v_{i}$ is attacked and removed from the network, the value of $E$ decreases, and the topological efficiency $\left[e\left(v_{i}\right)\right]$ is defined as:

$$
e\left(v_{i}\right)=e(O)-e\left(v_{i}^{\prime}\right)
$$

where $E(O)$ is the original topological efficiency of the network and $e\left(v^{\prime}{ }_{i}\right)$ is the network topological efficiency after $v_{i}$ being attacked. A higher value of $e\left(v_{i}\right)$ indicates the greater impact of $v_{i}$ on the network efficiency. In another words, $v_{i}$ is more crucial to topological reliability.

With passenger flow considered, the station vulnerability $\left[S\left(v_{i}\right)\right]$ is defined as:

$$
S\left(v_{i}\right)=R\left(v_{i}\right) \times p\left(v_{i}\right)
$$

where $R\left(v_{i}\right)$ is the reductions in network serviceability caused by the malfunction of $v_{i}$ and $p\left(v_{i}\right)$ is the probability that station $v_{i}$ is attacked and removed from the network. In this equation, $R\left(v_{i}\right)$ is relevant to $e\left(v_{i}\right)$ and passenger flow influence $\left(I_{i}\right)$, as defined in Section 3.4.2:

$$
R\left(v_{i}\right)=e\left(v_{i}\right) \times I_{i}
$$

The index reveals the reduction in network topological efficiency and the number of travelers suffering such a reduction when station $v_{i}$ is attacked.

\section{Case Study of Shanghai Metro}

\subsection{Basic Network Information}

In this section, the network and station vulnerabilities of Shanghai Metro were studied based on the proposed vulnerability model. By June 2014, Shanghai Metro had 287 nodes and 317 links, with an average degree of 2.2, which is at the middle level among urban rail transit networks in major cities throughout the world. Approximately $80 \%$ of the total number of nodes has a degree of two or above, while the nodes with a degree of four or above are approximately $9 \%$. Only one node has a degree of eight, and two other nodes have degrees and six and five, respectively, indicating that the nodes in Shanghai Metro seldom have a large degree. A detailed map of Shanghai Metro can be found in [28]

Table 1 presents the top ten important stations of Shanghai Metro based on node degree and betweenness, respectively. Century Avenue (No. 1) is the node with the largest degree of eight, which means that the station connects with the other eight stations within the network. Caoyang Road possesses the largest node betweenness of 19,114, indicating that overall, 19,114 shortest paths within the network pass the station. It is also worth noticing that the two rankings are quite different. Some stations, such as Caoyang Road and Zhenping Road, both with node degrees of four, possess higher node betweenness than Century Avenue and People's Square (with node degrees of eight and six, respectively). 
Table 1. Top ten important stations of Shanghai Metro.

\begin{tabular}{|c|c|c|c|c|c|}
\hline \multicolumn{3}{|c|}{ Station Ranking Based on Node Degree $\left(D_{i}\right)$} & \multicolumn{3}{|c|}{ Station Ranking Based on Node Betweenness $\left(B_{i}\right)$} \\
\hline No. & $D_{i}$ & Station Name & No. & $B_{i}$ & Station Name \\
\hline 1 & 8 & Century Avenue & 1 & 19,114 & Caoyang Road \\
\hline 2 & 6 & Xujiahui & 2 & 18,738 & Xujiahui \\
\hline 3 & 6 & People's Square & 3 & 18,566 & Century Avenue \\
\hline 4 & 5 & Oriental Sports Center & 4 & 18,180 & Zhenping Road \\
\hline 5 & 5 & Yishan Road & 5 & 17,848 & People's Square \\
\hline 6 & 4 & South Shaanxi Road & 6 & 17,574 & Shanghai Railway Station \\
\hline 7 & 4 & Changshu Road & 7 & 14,206 & Baoshan Road \\
\hline 8 & 4 & Shanghai Railway Station & 8 & 13,556 & Oriental Sports Center \\
\hline 9 & 4 & Shanghai Indoor Stadium & 9 & 12,868 & Hailun Road \\
\hline 10 & 4 & Zhaojiabang Road & 10 & 12,560 & Zhongtan Road \\
\hline
\end{tabular}

\subsection{Topological Vulnerability Analysis}

Figure 2 depicts the deterioration in network efficiency of Shanghai Metro under malicious attacks. The original network efficiency is 0.0852 , indicating a poor overall connectivity of the network. As can be seen from Figure 2, the random attacks cause minimal losses in network efficiency among the three malicious attacks. When $10 \%$ of the nodes (29 nodes) are attacked and removed from the network, the efficiency decreases by $37.6 \%$. However, both the largest degree node-based and the highest betweenness node-based attacks cause much larger losses in network efficiency. Under these two circumstance, when $10 \%$ of the nodes are removed, the network efficiencies decrease by $89.6 \%$ and $90 \%$, respectively. The highest betweenness node-based attacks cause slightly larger losses than the largest degree node-based attacks, but only a few differences exist in between. This means that stations with higher node betweenness have at least the same influence on network efficiency as stations with a larger node degree. These stations with higher betweenness may not be important from a common sense perspective, but they have a larger impact on maintaining network connectivity. It is also inferred that urban rail transit networks are vulnerable to the largest degree node-based attacks, as well as the highest betweenness node-based attacks, but are comparably rather robust to random attacks.

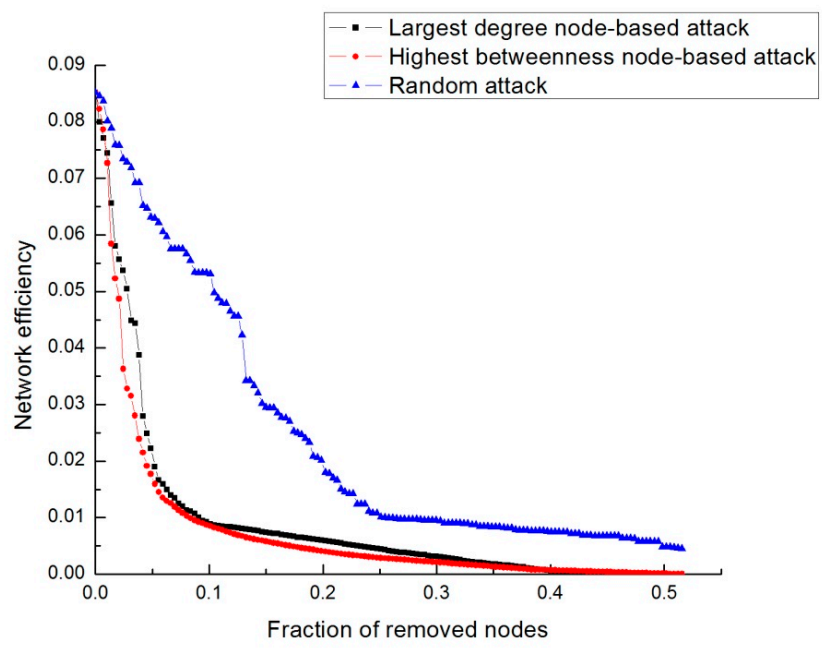

Figure 2. Network efficiency of Shanghai Metro under malicious attacks. 


\subsection{Functional Vulnerability Analysis}

Figure 3 depicts the changes in the network size of Shanghai Metro under malicious attacks. The largest degree node-based attack causes the maximum losses in the network size among the three malicious attacks. When $20 \%$ of the nodes (58 nodes) were attacked and removed from the network, the network size decreases by $39 \%$. Apart from the 58 attacked nodes, this also results in 54 isolated nodes in the network, indicating that the stations with a large node degree are generally important in maintaining network transport ability.

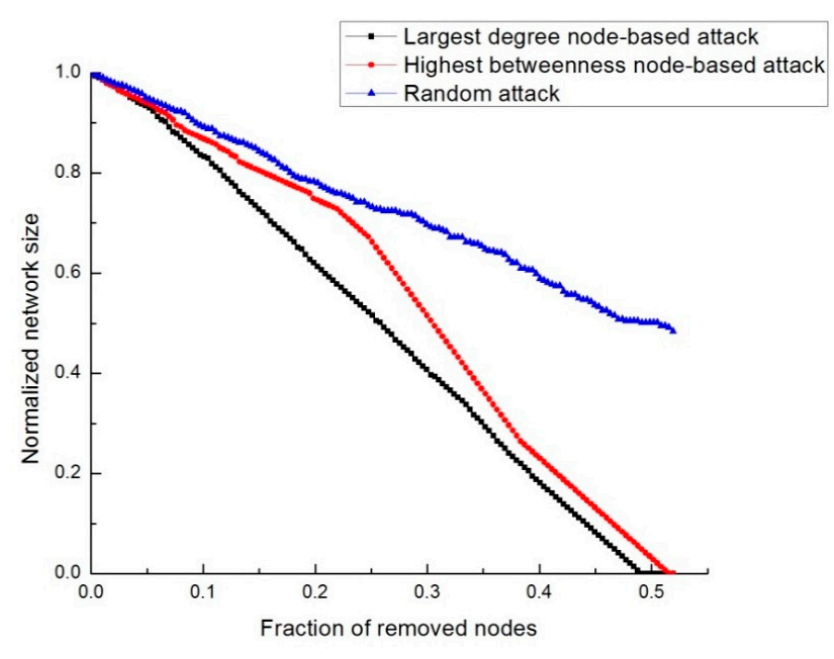

Figure 3. Network size of Shanghai Metro under malicious attacks.

Figure 4 depicts the changes in the connected OD ratio of Shanghai Metro under malicious attacks. As can be seen, malicious attacks have larger impacts on OD connectivity. When the top seven stations with the highest betweenness were attacked, the connected OD ratio decreases to only $29 \%$. Moreover, when $10 \%$ of the nodes ( 29 nodes) were attacked and removed from the network, the connected OD ratio decreases to approximately $2 \%$ under both the largest degree node-based and the highest betweenness nodebased attacks. Only 760 OD pairs are still connected out of 41,041 original pairs, and the network is almost paralyzed, demonstrating that OD connectivity is vulnerable to malicious attacks.

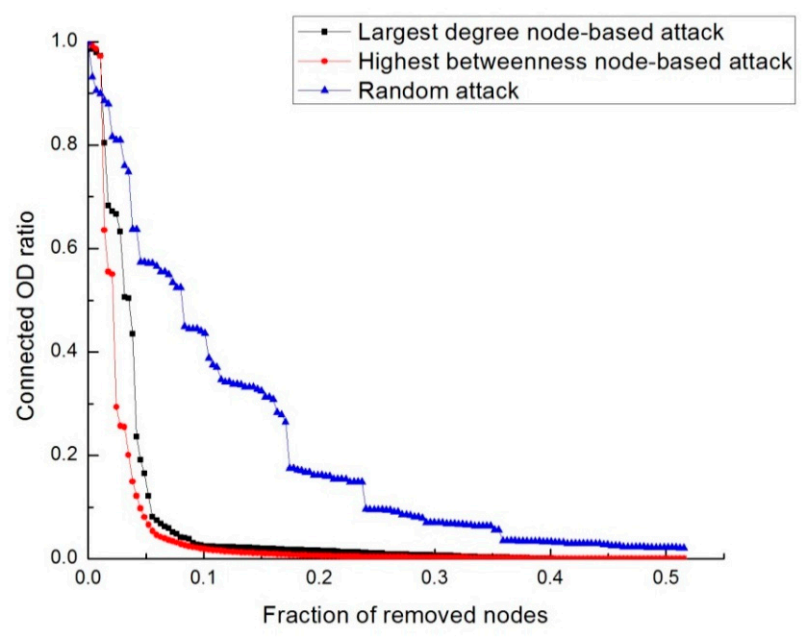

Figure 4. Connected origin-destination (OD) ratio of Shanghai Metro under malicious attacks. 


\subsection{Station Vulnerability Analysis}

\subsubsection{Calculations of Station Topological Efficiency}

Figure 5 depicts the topological efficiency $e\left(v_{i}\right)$ of each interchange station of Shanghai Metro. Only the top 20 stations are presented, because of space limitation, where Caoyang Road station and Oriental Sports Center station possess the largest station topological efficiency among the 41 interchange stations. When either of the two stations was attacked and removed from the network, the network topological efficiency decreases by $11.08 \%$.

To better illustrate the topological efficiencies of stations, six OD pairs with the highest demand volume of Shanghai Metro were selected and are presented in Table 2, accounting for approximately $13 \%$ of the total demand. With the shortest path algorithm, the fastest transit routes between these pairs were determined when the network is operated at full capacity. The selected routes may include one or several metro line(s), with some OD pairs relying on a single line and others with transfers. Ideal travel time, as listed in the last column of the table, includes transfer penalties.

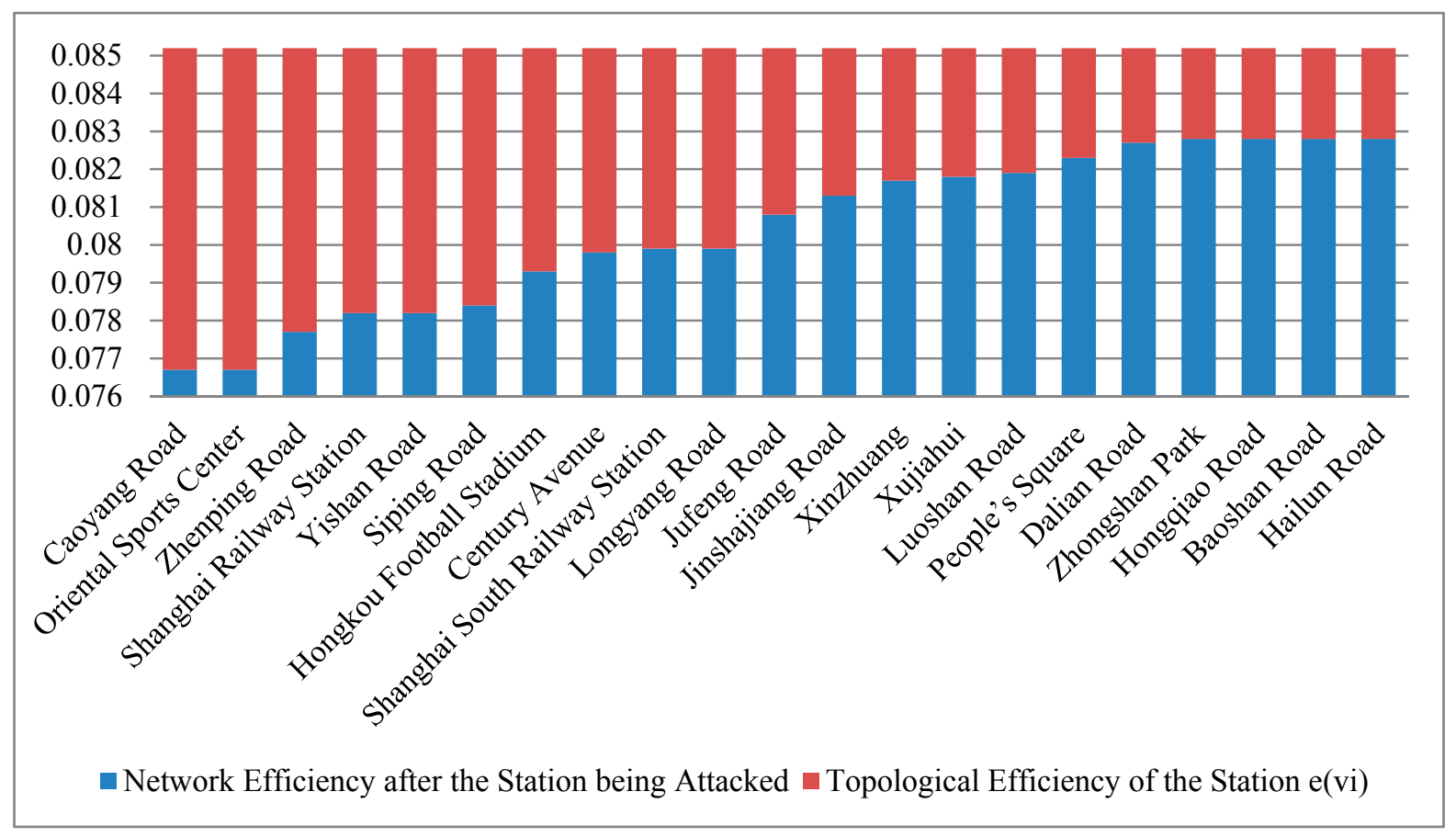

Figure 5. Topological efficiencies of the interchange stations of Shanghai Metro.

Table 2. Shortest travel time for selected OD pairs.

\begin{tabular}{|c|c|c|c|c|}
\hline \multirow{2}{*}{ No. } & \multicolumn{2}{|c|}{ OD Pair } & \multirow{2}{*}{ Route } & \multirow{2}{*}{$\begin{array}{c}\text { Ideal Travel } \\
\text { Time (min) }\end{array}$} \\
\hline & Origin & Destination & & \\
\hline 1 & Dongchuan Road & Lujiazui & Line 5-Line 1-Line 2 & 70 \\
\hline 2 & Zhongtan Road & Dongchang Road & Line 4-Line 1-Line 2 & 31 \\
\hline 3 & Gongkang Road & Shanghai South Railway Station & Line 1 & 51 \\
\hline 4 & Pengpu Xincun & Huamu Road & Line 1-Line 2-Line 7 & 58 \\
\hline 5 & Hongqiao Railway Station & Middle Yanggao Road & Line 2-Line 9 & 50 \\
\hline 6 & Yishan Road & Yuanshen Stadium & Line 9-Line 6 & 35 \\
\hline
\end{tabular}


Table 3 shows the new transit travel time between the selected ODs when specific transfer centers have been removed. The removed interchange nodes are Xujiahui, People's Square, Century Avenue and Shanghai Railway Station with topological efficiency $e\left(v_{i}\right)$ of 0.0034, 0.0029, 0.0054 and 0.0070, respectively. As can be inferred from Table 3, when a station with low topological efficiency, such as Xujiahui or People's Square, is out of service, locations remain accessible, but the travel time to those locations likely increases. This reveals that stations with lower topological efficiency may have an impact on the system travel time, but generally do not result in any unavailable nodes within the network. However, when a station with higher topological efficiency, such as Century Avenue or Shanghai Railway Station, is out of service, some stations become inaccessible by metro. Take Shanghai Railway Station as an example: when the node is removed, Routes 2, 3 and 4 significantly decline in connectivity. For Route 2, the travel time increases by $19.4 \%$, while Routes 3 and 4 become inaccessible.

Table 3. Shortest travel time for OD pairs after interchange station removal.

\begin{tabular}{cccccc}
\hline \multirow{2}{*}{$\begin{array}{c}\text { Route } \\
\text { No. }\end{array}$} & Ideal Travel & \multicolumn{5}{c}{ Travel Time with Station Removed (min) } \\
\cline { 3 - 6 } & Time (min) & Xujiahui & People's Square & Century Avenue & Shanghai Railway Station \\
\hline 1 & 70 & 81 & 78 & 70 & 70 \\
2 & 31 & 31 & 32 & 31 & 37 \\
3 & 51 & 59 & 59 & 51 & Not Accessible \\
4 & 58 & 58 & 63 & 73 & Not Accessible \\
5 & 50 & 50 & 64 & Not Accessible & 50 \\
6 & 35 & 41 & 35 & 91 & 35 \\
\hline Route & Ideal Travel & \multicolumn{5}{c}{ Percentage Change in Travel Time } \\
\cline { 3 - 6 } No. & Time (min) & Xujiahui & People's Square & Century Avenue & Shanghai Railway Station \\
\hline 1 & 70 & $15.7 \%$ & $11.4 \%$ & $0.0 \%$ & $0.0 \%$ \\
2 & 31 & $0.0 \%$ & $3.2 \%$ & $0.0 \%$ & $19.4 \%$ \\
3 & 51 & $15.7 \%$ & $15.7 \%$ & $0.0 \%$ & N/A \\
4 & 58 & $0.0 \%$ & $8.6 \%$ & $25.9 \%$ & N/A \\
5 & 50 & $0.0 \%$ & $28.0 \%$ & N/A & $0.0 \%$ \\
6 & 35 & $17.1 \%$ & $0.0 \%$ & $160.0 \%$ & $0.0 \%$ \\
\hline
\end{tabular}

\subsubsection{Calculation of Station Passenger Flow}

While urban rail transit networks are viewed as complex networks, what differentiates them from other complex networks (such as the power grid, etc.) is the interactions between infrastructures (stations, lines, etc.) and the passengers. Passenger flow is an important attribute of metro operations and also a critical factor for vulnerability analysis. Large passenger flow would add pressure to the network system and increase the probability of breakdown, and the passenger flow affected by the breakdown of a certain metro station also reveals the incident severity and the station importance. Therefore, two vulnerability indexes_-passenger flow influence $\left(I_{i}\right)$ and platform passenger flow $\left(P_{i}\right)$ - are defined to relate to the consequences and probability, respectively.

Passenger flow influence $\left(I_{i}\right)$ is defined as the flow volume that is affected by the breakdown of a certain station $v_{i}$, represented as:

$$
I_{i}=\sum O F_{i}+D F_{i}+P F_{i}
$$


where $O F_{i}$ is the passenger flow whose travel origins are $v_{i}, D F_{i}$ is the passenger flow whose travel destinations are $v_{i}$ and $P F_{i}$ is the passenger flow whose travel paths pass $v_{i}$. The index not only reflects the importance of the station $v_{i}$ in the urban rail transit network, but also reveals the consequences and severity of the station breakdown.

Platform passenger flow $\left(P_{i}\right)$ is defined as:

$$
P_{i}=\sum O F_{i}+D F_{i}+T F_{i}
$$

where $T F_{i}$ is the passenger flow that transfers at $v_{i} . P_{i}$ depicts the passenger volume at station $v_{i}$ during a certain period of time. Large platform passenger flow, especially common in peak hours, would add pressure to the platform operations and increase the probability of accidents.

While passengers may choose different routes for the same OD pair due to personal reasons, the problem is simplified by using the path matrix $\left\{l_{i j}\right\}$ obtained in Section 2.1 as the routes for given OD pairs. The route choice model provides the shortest path $\left(l_{i j}\right)$ between any two nodes $v_{i}$ and $v_{j}$ with metro interchange taken into considerations. In this model, the passenger flow-related indexes are calculated as:

$$
\begin{gathered}
O F_{i}=\sum_{j=1}^{N} F_{i j} \\
D F_{i}=\sum_{j=1}^{N} F_{j i} \\
P F_{i}=\sum_{v_{i} \subseteq l_{m n} \in\left\{l_{i j}\right\}, m, n \neq i} F_{m n} \\
T F_{i}=\sum_{v_{i}^{1}, v_{i}^{2} \subseteq l_{m m} \in\left\{l_{i j}\right\}, m, n \neq i} F_{m n}
\end{gathered}
$$

where $F_{i j}$ is the passenger flow for the OD pair $i$ to $j, N$ is the number of stations in the network and $v_{i}{ }^{1}$ and $v_{i}^{2}$ are any two virtual nodes within the node $v_{i}$. When the shortest paths for any OD pairs pass both $v_{i}{ }^{1}$ and $v_{i}{ }^{2}$, this means that passengers have to make a transfer at station $v_{i}$.

For validation purposes, field traffic data of Shanghai Metro were obtained from the Automatic Fare Collection (AFC) of Shanghai Shentong Metro Group Co., Ltd. Information, including entry station ID, exit station ID, passenger flow within a 5-min period and ticket types, was recorded, from which the OD matrix can be approximated. Then, passenger flow indexes were calculated and analyzed based on the OD matrix of 16 September 2013, from 7:30 a.m. to 8:30 a.m., the peak hour of Shanghai Metro for a typical weekday. A total of 370,414 raw records were obtained, and the enter and exit passenger flows at each station are supposed to be constant during peak hours.

Figure 6 presents the results of platform passenger flow $\left(P_{i}\right)$ and passenger flow influence $\left(I_{i}\right)$ of Shanghai Metro during peak hours in descending order of $P_{i}$. With space limited, only the top 20 stations were presented. As can be seen from Figure 6, the station with the largest platform passenger flow $\left(P_{i}\right)$ and passenger flow influence $\left(I_{i}\right)$ is People's Square. The platform passenger flow of this station is as high as 67,664 per hour, which brings large challenges to the station management and increases the difficulties in keeping order. Meanwhile, the passenger flow influence of People's Square Station is 135,481 per hour, indicating that more than 135 thousand passengers would be affected by the 
breakdown of this station during one hour. They either have to change to the routes with longer lengths on the metro or to choose other transport modes.

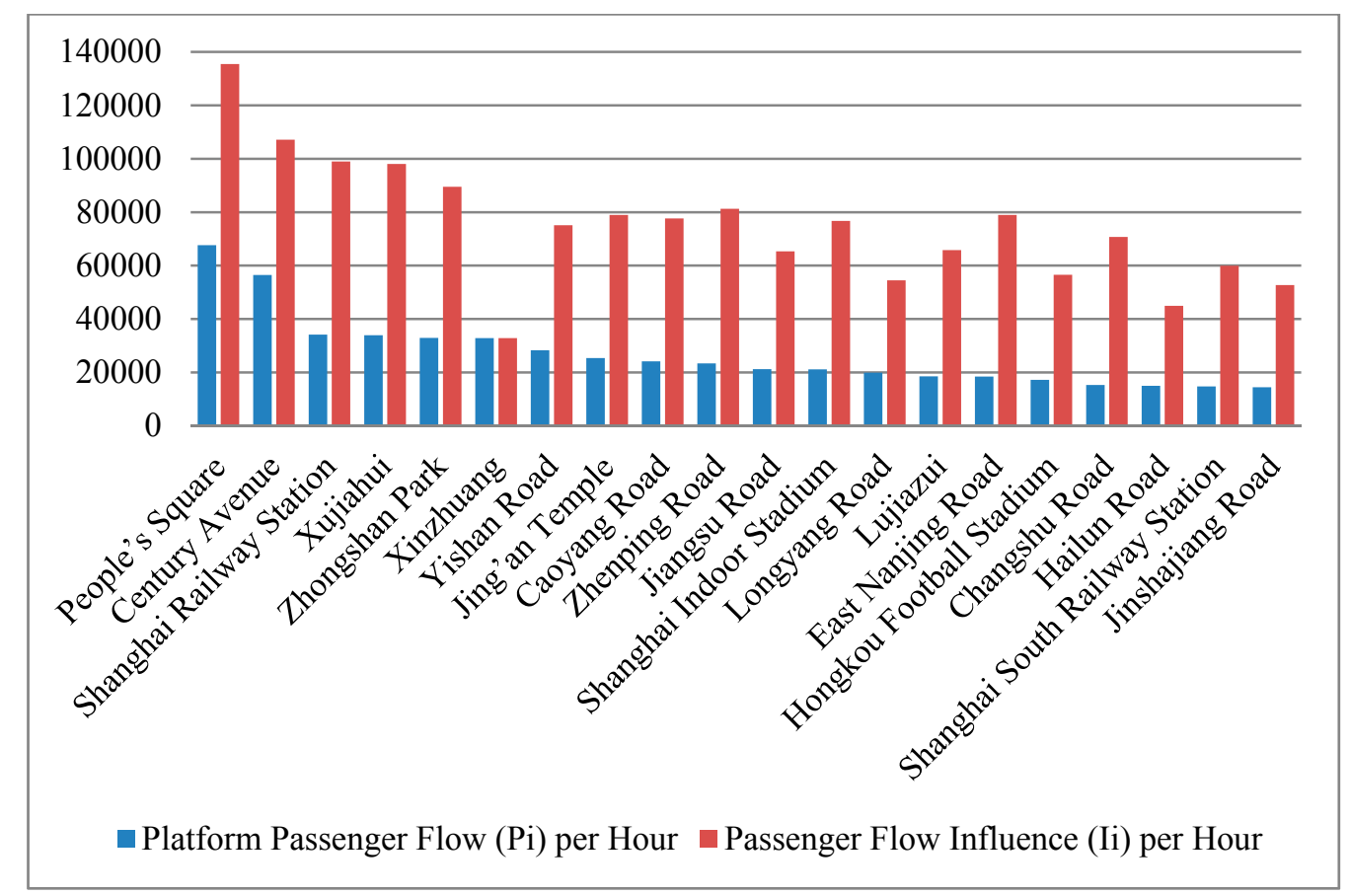

Figure 6. Top 20 heavy traffic stations of Shanghai Metro in peak hours.

\subsubsection{Station Vulnerability Evaluations}

Based on the field data, the station vulnerability of each station $v_{i}\left[S\left(v_{i}\right)\right]$ is further studied. As interchange stations are generally more important in maintaining network connectivity, the 41 interchange stations of Shanghai Metro were studied for peak hours. Table 4 presents the malfunction severity of these interchange stations. As shown in Table 4, Shanghai Railway Station ranks the first, which means the malfunction of this station causes the largest reduction of network connectivity. The topological efficiency of Shanghai Railway Station is 0.007 , and the breakdown would affect almost 99 thousand passengers within one hour. Both the reduction in network efficiency and the influence on passenger flow have an enormous impact on the entire network.

On the other hand, although People's Square Station has the largest passenger flow influence on the network, the topological efficiency of the station is approximately only one-third that of Caoyang Road Station. This means that while a large amount of passengers have to re-route due to the breakdown of People's Square Station, the alternative route choices do not decrease their travel efficiencies too much, and most can still arrive at their destinations within a certain duration with other alternative routes.

Oppositely, Oriental Sports Center Station ranks the first in station topological efficiency $e\left(v_{i}\right)$, but has a relatively small passenger flow influence $\left(I_{i}\right)$. This indicates that passengers generally have no other choices but to take much longer routes to their destinations during the breakdown of this station or, even worse, to switch to other transport modes. However, as the station is located in the suburbs of Shanghai, the passenger flow of the station is only about $20 \%$ that of People's Square. Consequently, not many passengers would be influenced by the breakdown of the station, and therefore, the overall reduction in network serviceability of this station ranks only 12 th among the 41 interchange stations of Shanghai Metro. 
Table 4. Malfunction severities of 41 interchange stations of Shanghai Metro.

\begin{tabular}{|c|c|c|c|c|c|c|c|}
\hline \multirow[t]{2}{*}{ Station Name } & \multirow[t]{2}{*}{$\begin{array}{c}\text { Degree } \\
\left(D_{i}\right)\end{array}$} & \multicolumn{2}{|c|}{$\begin{array}{l}\text { Topological } \\
\text { Efficiency of } \\
\text { Station } e\left(v_{i}\right) \\
\end{array}$} & \multicolumn{2}{|c|}{$\begin{array}{c}\text { Passenger Flow } \\
\text { Influence }\left(I_{i}\right) \\
\text { per Hour } \\
\end{array}$} & \multicolumn{2}{|c|}{$\begin{array}{c}\text { Reductions in } \\
\text { Network } \\
\text { Serviceability } R\left(v_{i}\right) \\
\end{array}$} \\
\hline & & $e\left(v_{i}\right)$ & No. & $\left(\mathbf{I}_{\mathbf{i}}\right)$ & No. & $\mathbf{R}\left(\mathbf{v}_{\mathbf{i}}\right)$ & No. \\
\hline Shanghai Railway Station & 4 & 0.007 & 4 & 98,963 & 3 & 692.74 & 1 \\
\hline Caoyang Road & 4 & 0.0085 & 1 & 77,687 & 9 & 660.34 & 2 \\
\hline Zhenping Road & 4 & 0.0075 & 3 & 81,264 & 6 & 609.48 & 3 \\
\hline Century Avenue & 8 & 0.0054 & 8 & 107,152 & 2 & 578.62 & 4 \\
\hline Yishan Road & 5 & 0.007 & 4 & 75,087 & 11 & 525.61 & 5 \\
\hline People’s Square & 6 & 0.0029 & 16 & 135,481 & 1 & 392.89 & 6 \\
\hline Hongkou Football Stadium & 4 & 0.0059 & 7 & 56,562 & 16 & 333.72 & 7 \\
\hline Xujiahui & 6 & 0.0034 & 14 & 98,013 & 4 & 333.24 & 8 \\
\hline Shanghai South Railway Station & 3 & 0.0053 & 9 & 59,934 & 14 & 317.65 & 9 \\
\hline Longyang Road & 4 & 0.0053 & 9 & 54,452 & 17 & 288.60 & 10 \\
\hline Siping Road & 4 & 0.0068 & 6 & 37,863 & 27 & 257.47 & 11 \\
\hline Oriental Sports Center & 5 & 0.0085 & 1 & 26,549 & 34 & 225.67 & 12 \\
\hline Zhongshan Park & 4 & 0.0024 & 18 & 89,514 & 5 & 214.83 & 13 \\
\hline Jinshajiang Road & 3 & 0.0039 & 12 & 52,750 & 20 & 205.73 & 14 \\
\hline East Nanjing Road & 4 & 0.0021 & 22 & 78,957 & 8 & 165.81 & 15 \\
\hline Jing'an Temple & 4 & 0.0019 & 26 & 78,968 & 7 & 150.04 & 16 \\
\hline Shanghai Indoor Stadium & 4 & 0.0019 & 26 & 76,716 & 10 & 145.76 & 17 \\
\hline Jiangsu Road & 4 & 0.002 & 24 & 65,339 & 13 & 130.68 & 18 \\
\hline Hongqiao Road & 4 & 0.0024 & 18 & 53,710 & 18 & 128.90 & 19 \\
\hline Baoshan Road & 3 & 0.0024 & 18 & 53,407 & 19 & 128.18 & 20 \\
\hline Xinzhuang & 2 & 0.0035 & 13 & 32,862 & 31 & 115.02 & 21 \\
\hline Zhongtan Road & 2 & 0.0021 & 22 & 51,724 & 21 & 108.62 & 22 \\
\hline Hailun Road & 4 & 0.0024 & 18 & 44,931 & 23 & 107.83 & 23 \\
\hline Changshu Road & 4 & 0.0015 & 34 & 70,721 & 12 & 106.08 & 24 \\
\hline Lujiabang Road & 4 & 0.002 & 24 & 48,090 & 22 & 96.18 & 25 \\
\hline South Shaanxi Road & 4 & 0.0014 & 37 & 59,742 & 15 & 83.64 & 26 \\
\hline Zhaojiabang Road & 4 & 0.0017 & 31 & 44,621 & 24 & 75.86 & 27 \\
\hline South Xizang Road & 4 & 0.0019 & 26 & 39,224 & 26 & 74.53 & 28 \\
\hline Dalian Road & 4 & 0.0025 & 17 & 29,277 & 32 & 73.19 & 29 \\
\hline Yaohua Road & 4 & 0.0019 & 26 & 37,044 & 28 & 70.38 & 30 \\
\hline Dongan Road & 4 & 0.0017 & 31 & 35,465 & 29 & 60.29 & 31 \\
\hline Laoximen & 4 & 0.0017 & 31 & 32,975 & 30 & 56.06 & 32 \\
\hline Jiaotong University & 4 & 0.0018 & 30 & 29,017 & 33 & 52.23 & 33 \\
\hline Jufeng Road & 4 & 0.0044 & 11 & 11,105 & 40 & 48.86 & 34 \\
\hline West Yan'an Road & 2 & 0.0011 & 40 & 41,791 & 25 & 45.97 & 35 \\
\hline Lancun Road & 4 & 0.0013 & 38 & 24,707 & 35 & 32.12 & 36 \\
\hline West Gaoke Road & 4 & 0.0015 & 34 & 21,295 & 36 & 31.94 & 37 \\
\hline Hongqiao Airport T2 & 3 & 0.0015 & 34 & 19,667 & 37 & 29.50 & 38 \\
\hline Tiantong Road & 3 & 0.0012 & 39 & 13,359 & 39 & 16.03 & 39 \\
\hline Hongqiao Railway Station & 2 & 0.0009 & 41 & 15,798 & 38 & 14.22 & 40 \\
\hline Luoshan Road & 2 & 0.0033 & 15 & 619 & 41 & 2.04 & 41 \\
\hline
\end{tabular}


As shown in Table 4, the malfunction severities of interchange stations differ greatly. Some stations may not be as important as others. For example, stations, such as Lancun Road or West Gaoke Road, have an $R\left(v_{i}\right)$ of only $4 \%$ that of Shanghai Railway Station. To this end, it is concluded that the vulnerability model assists with distinguishing critical stations within the network from a systematic perspective.

The consequences of station malfunctions were analyzed, as shown in Table 5. Platform passenger flow $\left(P_{i}\right)$ refers to taking the probability of breakdown into consideration. While it is generally difficult to predict the probabilities of certain events, such as terrorist actions and extreme weather, etc., the probability of a station breakdown $p\left(v_{i}\right)$ was found positively correlated with $P_{i}[4,22]$. Although the two parameters do not have strictly linear correlations, a large $P_{i}$, especially common in peak hours, would add pressure to the platform operations and increase the probability of station breakdown.

Table 5. Station vulnerabilities of critical stations of Shanghai Metro.

\begin{tabular}{clcccc}
\hline No. & Station Name & $\begin{array}{c}\text { Reductions in } \\
\text { Network } \\
\text { Serviceability } \\
\boldsymbol{R}\left(\boldsymbol{v}_{\boldsymbol{i}}\right)\end{array}$ & $\begin{array}{c}\text { Platform } \\
\text { Passenger Flow } \\
\left(\boldsymbol{P}_{\boldsymbol{i}}\right) \text { per Hour }\end{array}$ & $\begin{array}{c}\text { Estimated } \\
\text { Breakdown } \\
\text { Probability due to } \\
\text { Large Flow } \boldsymbol{p}\left(\boldsymbol{v}_{\boldsymbol{i}}\right)\end{array}$ & $\begin{array}{c}\text { Station } \\
\text { Vulnerability } \\
\boldsymbol{S}\left(\boldsymbol{v}_{\boldsymbol{i}}\right)\end{array}$ \\
\hline 1 & Century Avenue & 578.62 & 56,461 & $5.2 \%$ & 30.2 \\
2 & People's Square & 392.89 & 67,664 & $6.3 \%$ & 24.6 \\
3 & Shanghai Railway Station & 692.74 & 34,148 & $3.2 \%$ & 21.9 \\
4 & Caoyang Road & 660.34 & 24,170 & $2.2 \%$ & 14.8 \\
5 & Yishan Road & 525.61 & 28,302 & $2.6 \%$ & 13.8 \\
6 & Zhenping Road & 609.48 & 23,354 & $2.2 \%$ & 13.2 \\
7 & Xujiahui & 333.24 & 33,960 & $3.1 \%$ & 10.5 \\
8 & Hongkou Football Stadium & 333.72 & 17,211 & $1.6 \%$ & 5.3 \\
9 & Longyang Road & 288.6 & 19,891 & $1.8 \%$ & 5.3 \\
10 & Shanghai South Railway Station & 317.65 & 14,760 & $1.4 \%$ & 4.3 \\
\hline
\end{tabular}

Table 5 presents the top 10 critical interchange stations of Shanghai Metro with the largest station vulnerability. As can be seen, the platform passenger flows of stations, such as People's Square, Century Avenue, Shanghai Railway Station and Xujiahui, are extremely enormous during peak hours. Such pressures on the station operation and management caused by huge passenger flows are turned into increases in the probabilities of sudden events and station breakdown. Estimated breakdown probabilities are therefore calculated as the ratio of $P_{i}$ to the overall passenger flow of Shanghai Metro during a.m. peak hours (1,081,218 passengers per hour). An overall consideration of the consequences and probabilities of station breakdown brings the conclusion that Century Avenue, People's Square and Shanghai Railway Station are the three most critical stations of Shanghai Metro in maintaining network serviceability. These stations are vulnerable to attacks due to large passenger flow, and the consequences of breakdown are severe and, therefore, need to be better protected.

The results of the calculations are summarized and illustrated in Figure 7. The locations of the top ten stations in terms of topological efficiency $\left(e\left(v_{i}\right)\right)$, platform passenger flow $\left(P_{i}\right)$ and station vulnerability $\left(S\left(v_{i}\right)\right.$ ) (with specific data provided in Figures 5 and 6 and Table 5, respectively) are simultaneously marked on the Shanghai Metro map. As shown, the most critical stations in maintaining system serviceability are not necessarily the locations with the highest passenger throughput or the largest 
structural connectivity. It may be misleading or not appropriate if the greatest attention were paid to the busiest stations or the topologically important stations. Therefore, the evaluation model proposed in this paper proves to be a useful tool to assist traffic administrators with figuring out any neglected, but important stations in urban rail transit networks, so that priorities in funding and other resources could be allocated to the appropriate nodes efficiently.

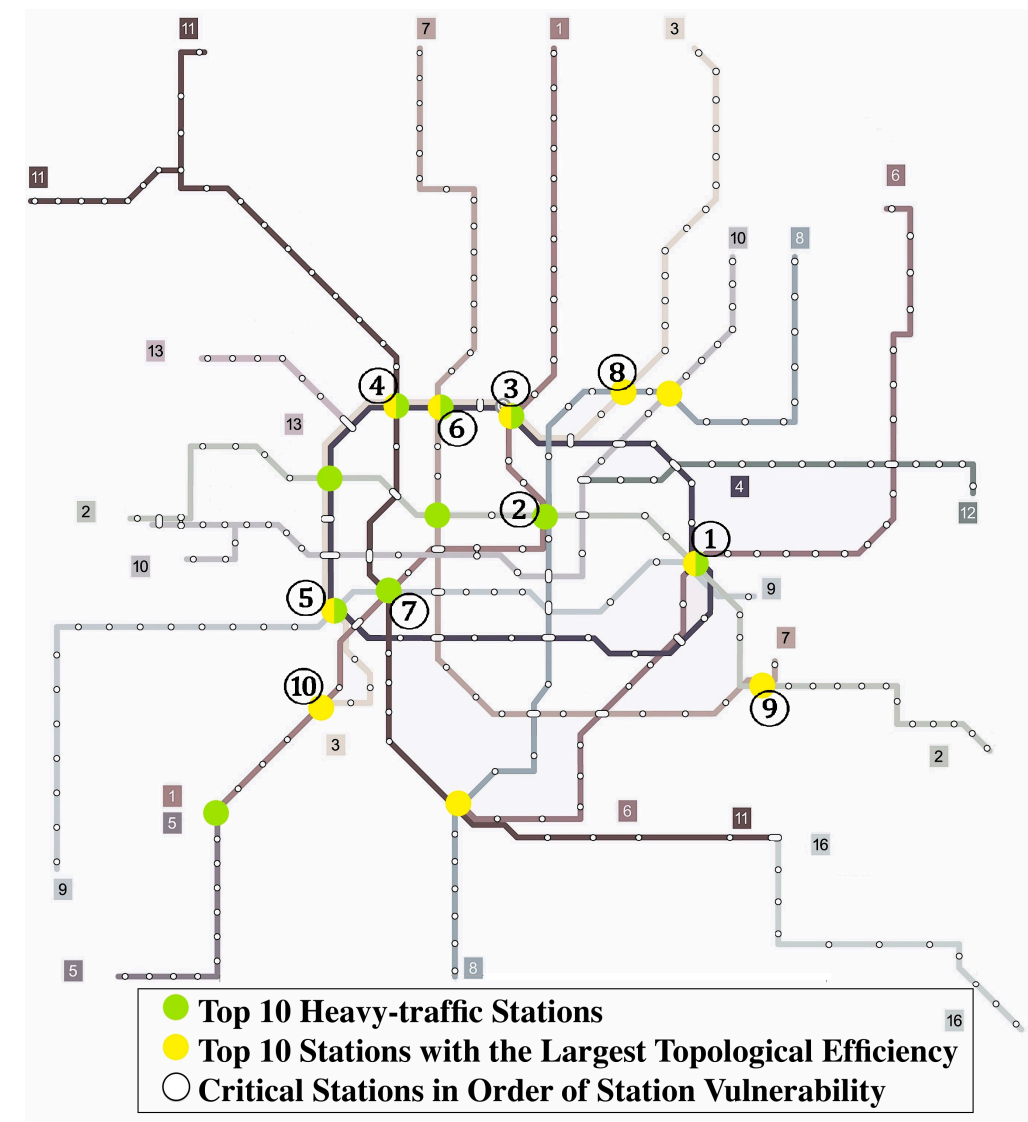

Figure 7. Locations of critical stations of Shanghai Metro.

\section{Conclusions}

This paper proposes a systematic framework to investigate the network and station vulnerabilities of urban rail transit networks. A vulnerability evaluation model is developed, optimized and verified. Special characteristics of urban rail transit networks, such as metro interchange and traveler route-choice habits, were taken into consideration. Field passenger flow was introduced and quantified in the vulnerability analyses. The probabilities of network malfunctions were then evaluated, and the incident severity and station importance were calculated.

Results from the case study of Shanghai Metro indicate that urban rail transit networks are generally vulnerable to the largest degree node-based attacks and the highest betweenness node-based attacks. Disruptions to a single station, especially intended terrorism attacks, would have severe impacts on the entire network. Furthermore, stations with the most significant effects on system connectivity are not necessarily locations with the largest passenger flow. A comprehensive evaluation model, as proposed in this paper, is therefore needed to accurately assess station vulnerability, so that attention could be provided to the most critical stations. 
The systematic framework of this paper is useful for transit system planners in terms of urban rail transit network design. Vulnerabilities of different urban rail transit networks could be analyzed and compared based on the model, and a reliable network geometry could then be obtained. Such a tool can provide theoretical support to the design and optimization of network layout and be used to explore possible improvements to network reliability when new lines are built [29].

Moreover, the case study has demonstrated the utility of the proposed vulnerability evaluation model in a real-world rail transit network. Crucial stations were identified based on the analyses, so that measurements to reduce incident possibilities or action plans to better respond to emergencies at these stations can be explored beforehand. The vulnerability model provides a proactive approach that assists with identifying network weaknesses and prepares for extreme events beforehand, rather than reacting afterward [30].

Further studies on the probabilities of network malfunctions may be conducted with additional factors considered. The interdependence of metro network with other transport modes can be investigated, so as to incorporate a wider range of travel behaviors into the vulnerability analysis. Alternative solutions to disruptions of urban rail transit networks may also be explored.

\section{Acknowledgments}

The authors would like to express their appreciation to Dr. Wei Zhu from School of Transportation Engineering, Tongji University for his valuable suggestions and assistance in this study. The research was sponsored in part by the National Natural Science Foundation of China (No. 71101109), the Key Project of Shanghai Soft Science Research Program (No. 14692106400), and the Chuntsung Project of Shanghai Jiao Tong University (No. 2013-3-01). Any opinions, findings and conclusions or recommendations expressed in this paper are those of the authors and do not necessarily reflect the views of the sponsors.

\section{Author Contributions}

Daniel (Jian) Sun and Yuhan Zhao conceived the project and wrote the manuscript together. Daniel (Jian) Sun mainly obtained the traveler IC card data, proposed the data analysis method, and suggested to introduce the passenger IC card data into the study. Yuhan Zhao analyzed the network data and passenger IC card data, carried out the vulnerability evaluation model, and performed the station vulnerability analysis using Shanghai Metro data. Qing-Chang Lu provided critical suggestions and inputs for the case study and helped with writing the manuscript. All authors have read and approved the final manuscript.

\section{Conflicts of Interest}

The authors declare that there is no conflict of interests regarding the publication of this paper.

\section{References}

1. Litman, T. Impacts of rail transit on the performance of a transportation system. Transp. Res. Record 2005, 1930, 21-29. 
2. Nguyen, T.P.K.; Beugin, J.; Marais, J. Method for evaluating an extended Fault Tree to analyze the dependability of complex systems: Application to a satellite-based railway system. Reliab. Eng. Syst. Saf. 2015, 133, 300-313.

3. Vuchic, V.R. Urban Transit: Operations, Planning, and Economics; Wiley: Hoboken, NJ, USA, 2005.

4. Beroggi, G.E. Integrated safety planning for underground systems. J. Hazard. Mater. 2000, 71, 17-34.

5. Canós, J.H.; de Zulueta, F. Using hypermedia to improve safety in underground metropolitan transportation. Multimedia Tools Appl. 2004, 22, 75-87.

6. Gabay, D. Fire safety: A short history in the Paris subway. Tunnel. Undergr. Space Technol. 2002, 17, 139-143.

7. Liao, F.; Arentze, T.; Timmermans, H. Multi-state supernetworks: Recent progress and prospects. J. Traffic Transp. Eng. (Engl. Ed.) 2014, 1, 13-27.

8. Del Río, P.M.S.; Hernández, J.A.; Aracil, J.; de Vergara, J.E.L.; Domżał, J.; Wójcik, R.; Duque, R. A reliability analysis of double-ring topologies with dual attachment using p-cycles for optical metro networks. Comput. Netw. 2010, 54, 1328-1341.

9. Derrible, S.; Kennedy, C. The complexity and robustness of metro networks. Physica A 2010, 389, 3678-3691.

10. De-Los-Santos, A.; Laporte, G.; Mesa, J.A.; Perea, F. Evaluating passenger robustness in a rail transit network. Transp. Res. Part C 2012, 20, 34-46.

11. Cadarso, L.; Marín, Á.; Maróti, G. Recovery of disruptions in rapid transit networks. Transp. Res. Part E 2013, 53, 15-33.

12. Berdica, K. An introduction to road vulnerability: What has been done, is done and should be done. Transp. Policy 2002, 9, 117-127.

13. Jenelius, E.; Petersen, T.; Mattsson, L.G. Importance and exposure in road network vulnerability analysis. Transp. Res. Part A 2006, 40, 537-560.

14. Bell, M.G.; Kanturska, U.; Schmöcker, J.D.; Fonzone, A. Attacker-defender models and road network vulnerability. Philos. Trans. R. Soc. A 2008, 366, 1893-1906.

15. Taylor, M.A.; Sekhar, S.V.; D'Este, G.M. Application of accessibility based methods for vulnerability analysis of strategic road networks. Netw. Spat. Econ. 2006, 6, 267-291.

16. Jenelius, E. Network structure and travel patterns: Explaining the geographical disparities of road network vulnerability. J. Transp. Geogr. 2009, 17, 234-244.

17. Gao, J.; Shi, Q. Definition and evaluation modeling of metro network invulnerability. J. China Railw. Soc. 2007, 29, 29-33. (In Chinese)

18. Wang, Y. Research on Connectivity Reliability of Urban Transit Network Based on Theory of Complex Network. Master's Thesis, Beijing Jiao Tong University, Beijing, 2008.

19. Zhang, J.; Xu, X.; Hong, L.; Wang, S.; Fei, Q. Networked analysis of the Shanghai subway network, in China. Physica A 2011, 390, 4562-4570.

20. Quan, R.; Liu, M.; Zhang, L. Vulnerability assessment of rainstorm water-logging in subway of Shanghai. Yangtze River 2011, 42, 13-17.

21. Han, Y.; Cheng, H.; Zhao, X.; Xue, X. Theoretic structure of urban mass transit operation safety based on vulnerability. Urban Mass Transit 2012, 15, 15-19.

22. Yuan, J.; Li, Q.; Jia, R.; Wang, Z. Analysis of operation vulnerabilities of urban metro network system. China Saf. Sci. J. 2012, 22, 92-98. 
23. Barabási, A.L.; Albert, R. Emergence of scaling in random networks. Science 1999, 286, 509-512.

24. Angeloudis, P.; Fisk, D. Large subway systems as complex networks. Physica A 2006, 367, 553-558.

25. Raveau, S.; Muñoz, J.C.; de Grange, L. A topological route choice model for metro. Transp. Res. Part A 2011, 45, 138-147.

26. Floyd, R.W. Algorithm 97: Shortest path. Commun. ACM 1962, 5, doi:10.1145/367766.368168.

27. Zhang, J.; Zhao, M.; Liu, H.; Xu, X. Networked characteristics of the urban rail transit networks. Physica A 2013, 392, 1538-1546.

28. Shanghai Metro Group Co., Ltd., Shanghai Metro Network Map. Available online: http://service.shmetro.com/yxxlt/index.htm (accessed on 2 January 2014).

29. Sun, D.J.; Zhang, C.; Zhang, L.; Chen, F.; Peng, Z.-R. Urban travel behavior analysis and travel time predicting based on floating car data. Transp. Lett. Int. J. Transp. Res. 2014, 6, 118-125.

30. Sun, D.J.; Ni, X.-Y.; Peng, Z.-R. An improved incremental assignment model for parking variable message sign location problem. J. Adv. Transp. 2015, doi:10.1002/atr.1305.

(C) 2015 by the authors; licensee MDPI, Basel, Switzerland. This article is an open access article distributed under the terms and conditions of the Creative Commons Attribution license (http://creativecommons.org/licenses/by/4.0/). 